\title{
Clinical Guideline Highlights for the Hospitalist: Secondary Fracture Prevention for Hospitalized Patients
}

\author{
Evan Siau, MD ${ }^{1,2}$, Matthew Harrington, MD ${ }^{1,2}$, Daniel Steinberg, MD ${ }^{1,2}$, Alfred Burger, MD, MS ${ }^{1,2 \star}$
}

${ }^{1}$ Department of Medicine, Icahn School of Medicine at Mount Sinai, New York, New York; ${ }^{2}$ Division of Hospital Medicine, Mount Sinai Beth Israel, New York, New York.

GUIDELINE TITLE: Secondary Fracture Prevention: Consensus Clinical Recommendations from a Multistakeholder Coalition

RELEASE DATE: September 20, 2019

PRIOR VERSION: Not applicable

DEVELOPER: American Society for Bone and Mineral Research Task Force and Multistakeholder Coalition
FUNDING SOURCE: American Society for Bone and Mineral Research and the Center for Medical Technology Policy

TARGET POPULATION: Adults 65 years or older with a hip or vertebral fracture. This review will focus on the core recommendations and their application in the practice of hospital medicine.

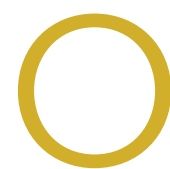

steoporosis is the most prevalent bone disease and a leading cause of morbidity and mortality in older people. According to the National Health and Nutrition Examination Survey, from 2005-2010, there were an estimated 10.2 million adults 50 years and older with osteoporosis and 43.4 million more with low bone mass in the United States. ${ }^{1}$ Osteoporotic fracture is a leading cause of hospitalization in the United States for women 55 years or older, ahead of heart attacks, stroke, and breast cancer. ${ }^{2}$ Despite elucidation of the pathogenesis of osteoporosis and the advent of effective and widely available therapies, a "treatment gap" separates the many patients who warrant therapy from the few who receive it. Systematic improvement strategies, such as coordinator-based fracture liaison services, have had a positive impact on addressing this treatment gap. ${ }^{3}$ There is an opportunity for hospitalists to further narrow this treatment gap.

The American Society of Bone and Mineral Research, in conjunction with the Center for Medical Technology Policy, developed consensus clinical recommendations to address secondary fracture prevention for people 65 years or older who have experienced a hip or vertebral fracture. ${ }^{4}$ We address six of the fundamental and two of the supplemental recommendations as they apply to the practice of hospital medicine.

\section{KEY RECOMMENDATIONS FOR HOSPITALISTS}

\section{Recommendations 1 and 2}

Communicate key information to the patient and their usual healthcare provider. Patients 65 years or older with a hip or vertebral fracture likely have osteoporosis and are at high risk for subsequent fractures, which can lead to a decline in func-

*Corresponding Author: Alfred Burger, MD, MS;

Email: alfred.burger@mountsinai.org; Telephone: 212-420-3369.

Published online first January 20, 2021.

Received: August 13, 2020; Revised: October 23, 2020;

Accepted: October 25, 2020

() 2021 Society of Hospital Medicine DOI 10.12788/jhm.3554 tion and an increase in mortality. Patients must be counseled regarding their diagnosis, their risks, and the actions they can take to manage their disease. Primary care providers must be notified of the occurrence of the fracture, the diagnosis of osteoporosis, and the plans for management.

We recommend hospitalists act as leading advocates for atrisk patients to ensure that this communication occurs during hospitalization. We encourage hospitals and institutions to adopt systematic interventions to facilitate postdischarge care for these patients. These may include implementing a fracture liaison service, with multidisciplinary secondary fracture-prevention strategies using physicians, pharmacists, nurses, social workers, and case managers for care coordination and treatment initiation.

Elderly patients with osteoporotic fragility fractures are at risk for further morbidity and mortality. Coordination of care between the inpatient care team and the primary care provider is necessary to reduce this risk. In addition to verbal communication and especially when verbal communication is not feasible, discharge documents provided to patients and outpatient providers should clearly identify the occurrence of a hip or vertebral fracture and a discharge diagnosis of osteoporosis if not previously documented, regardless of bone mineral density (BMD) results or lack of testing.

\section{Recommendation 3}

Regularly assess fall risk. Patients 65 years or older with a current or prior hip or vertebral fracture must be regularly assessed for risk of falls. Hospitalists can assess patients' ongoing risk for falls at time of admission or during hospitalization. Risk factors include prior falls; advanced age; visual, auditory, or cognitive impairment; decreased muscle strength; gait and balance impairment; diabetes mellitus; use of multiple medications, and others. ${ }^{5}$ Specialist evaluation by a physical therapist or a physiatrist should be considered. Active medications should be reviewed for adverse effects and interactions. The use of diuretics, antipsychotics, antidepressants, benzodiazepines, antiepileptics, and opioids should be minimized. 
Recommendations 4, 5, 6, and 11

Offer pharmacologic therapy and initiate calcium and vitamin $D$ supplementation. Recommendations 4 through 6 and 11 advocate pharmacologic interventions including bisphosphonates, denosumab, vitamin $D$, and/or calcium to reduce the risk of future fractures. Bisphosphonates are the cornerstone of pharmacologic therapy for secondary fracture prevention. The efficacy of these agents for prevention of subsequent fractures outweighs the potential for interference in healing of surgically repaired bones. ${ }^{6}$ Oral bisphosphonate therapy should be initiated in the hospital or at discharge. Parenteral bisphosphonates and denosumab may be utilized in patients unable to tolerate or absorb oral bisphosphonates due to esophageal or other gastrointestinal disease. Initiation of these agents should be delayed until after vitamin $D$ and calcium supplementation have been administered for 2 weeks after the fracture to reduce the risk of precipitating hypocalcemia, and they should not be used in patients with confirmed hypocalcemia until that is resolved. BMD measurement is not necessary prior to pharmacologic therapy initiation because the risk of fracture is elevated for these patients regardless of BMD. Patients without significant dental disease or planned oral or maxillofacial procedures may begin bisphosphonate therapy prior to a full dental assessment because risk of osteonecrosis of the jaw is low.

The guidelines recommend people 65 years or older with a hip or vertebral fracture receive daily supplementation of at least $800 \mathrm{IU}$ vitamin D. Patients unable to achieve an intake of 1,200 $\mathrm{mg} /$ day of calcium from food sources should receive daily calcium supplementation. The effect of vitamin D monotherapy on fracture risk is not clear; however, strong evidence suggests that fracture risk is reduced when individuals at high risk of deficiency receive supplementation with vitamin D and calcium. Calcium supplementation alone has not demonstrated reduction in fracture risk. Total daily calcium intake above 1,500 mg has not been shown to provide additional benefit and is potentially harmful.

\section{Recommendation 9}

Counsel patients on lifestyle modifications and consider physical therapy. Tobacco has a deleterious effect on bone density and increases risk for osteoporotic fragility fracture. ${ }^{7}$ Hospitalists should obtain tobacco use history from all patients with an osteoporotic fracture and provide tobacco cessation counseling when appropriate. Excessive alcohol consumption increases the risk of fall injuries. ${ }^{8}$ Hospitalists should counsel patients to limit alcohol intake to a maximum of two drinks a day for men and one drink a day for women.

Weight-bearing and strength-training exercises, particularly those involving balance and trunk muscle strength, are associated with reduction in fall-risk. Exercise must be tailored to the patient's physical capacity. Hospitalists may partner with physical therapists or physiatrists to facilitate development of an exercise plan to maximize benefit and minimize risk of injury.

\section{CRITIQUE}

We found this document to be highly informative and well cited, with ample evidence to support the recommendations.

\section{Methods in Preparing Guidelines}

The multistakeholder coalition did not employ a rigorous and standardized methodology for the guideline, such as GRADE (Grading of Recommendations Assessment, Development, and Evaluation); hence, no assessment of evidence quality, benefits and harms of an intervention, or resource use was provided.

\section{Potential Conflicts for Guideline Authors}

Eight guideline authors have pharmaceutical relationships with the manufacturer of one of the medications listed on the guidelines (Amgen-denosumab, Novartis-zoledronic acid). There are no disclosures reported from the multistakeholder coalition members who are not listed as guideline authors.

\section{AREAS IN NEED OF FUTURE STUDY}

We anticipate future studies may report outcomes focused on secondary prevention of fractures. Additionally, we would like to see new studies investigating patient-centered outcomes such as improvement in functional status and ambulatory independence based on improved postfracture medical therapies. We see an opportunity for studies assessing real-world outcomes to inform future recommendations, particularly after widespread implementation of secondary fracture prevention therapy either initiated during hospitalization or purposefully planned for after discharge.

We would like to see more trial data comparing the safety and cost-effectiveness of first-line therapy, namely oral bisphosphonates, to alternative treatments, particularly parenteral agents, which may improve treatment compliance because of the convenience in dosing frequency.

Disclosures: The authors reported having nothing to disclose.

\section{References}

1. Wright NC, Looker AC, Saag KG, et al. The recent prevalence of osteoporosis and low bone mass in the United States based on bone mineral density at the femoral neck or lumbar spine. J Bone Miner Res. 2014;29(11):2520-2526. https://doi.org/10.1002/jbmr.2269

2. Singer $A$, Exuzides $A$, Spangler $L$, et al. Burden of illness for osteoporotic fractures compared with other serious diseases among postmenopausal women in the United States. Mayo Clin Proc. 2015;90(1):53-62. https://doi. org/10.1016/j.mayocp.2014.09.011

3. McLellan AR, Gallacher SJ, Fraser M, McQuillian C. The fracture liaison service: success of a program for the evaluation and management of patients with osteoporotic fracture. Osteoporos Int. 2003;14(12):1028-1034. https:// doi.org/10.1007/s00198-003-1507-z

4. Conley RB, Adib G, Adler RA, et al. Secondary fracture prevention: consensus clinical recommendations from a multistakeholder coalition. J Bone Miner Res. 2020;35(1):36-52. https://doi.org/10.1002/jbmr.3877

5. Bueno-Cavanillas A, Padilla-Ruiz F, Jiménez-Moleón JJ, Peinado-Alonso CA, Gálvez-Vargas R. Risk factors in falls among the elderly according to extrinsic and intrinsic precipitating causes. Eur J Epidemiol. 2000;16(9):849-859. https://doi.org/10.1023/a:1007636531965

6. Vannucci L, Brandi ML. Healing of the bone with anti-fracture drugs. Expert Opin Pharmacother. 2016;17(17):2267-2272. https://doi.org/10.1080/146565 66.2016.1241765

7. Law MR, Hackshaw AK. A meta-analysis of cigarette smoking, bone mineral density and risk of hip fracture: recognition of a major effect. BMJ. 1997;315(7112):841-846. https://doi.org/10.1136/bmj.315.7112.841

8. Chen $\mathrm{CM}$, Yoon $\mathrm{YH}$. Usual alcohol consumption and risks for nonfatal fall injuries in the United States: results from the 2004-2013 National Health Interview Survey. Subst Use Misuse. 2017;52(9):1120-1132. https://doi.org/10.1 080/10826084.2017.1293101 